\title{
Optical Nonlinearity of Liquid Crystals in the Presence of Chained Ferroelectric Nanoparticles
}

\author{
J.B. POURSAmAD* AND M. EMdAdi \\ Department of Laser and Optical Engineering, University of Bonab, Bonab, Iran \\ (Received May 19, 2018; in final form December 31, 2018)
}

\begin{abstract}
A nematic liquid crystal cell doped with spherical ferroelectric nanoparticles is studied in the high frequency regime. Optical permittivity of the host nematic liquid crystal in the presence of chained ferroelectric nanoparticles, birefringence and optical Freedericksz transition threshold of the suspension are discussed theoretically. The director reorientation of doped nematic liquid crystal is due to the simultaneous presence of the oblique incidence of a light beam and external applied magnetic field in the small director reorientation approximation. Also, optical nonlinearity of the medium is analytically calculated here. The obtained optical nonlinearity is in the range of supra-nonlinearity.
\end{abstract}

DOI: 10.12693/APhysPolA.136.861

PACS/topics: 78.67.Bf, 77.84.--s

\section{Introduction}

Liquid crystals (LCs) are mesophases between the isotropic liquids and the solid crystals. They can flow as fluids and have some characteristics of solids as optical properties [1]. Their flexibility in the presence of electric and magnetic fields makes them a potential candidate for scientific investigations and industrial applications [2]. Due to the slow orientational response time of LCs, it was conceived that they cannot respond to the optical fields, whereas, it was shown theoretically and experimentally that LCs reorient in the presence of an optical field [3-8]. It was shown that the response of the LCs to the optical fields increase in the presence of proper dopants $[9,10]$. Light induced thermal [11-13], conformational [14], heat-flow [15, 16], surface-driven [17-21], thermomechanical [22-24], and photorefractive [25] effects give rise to large optical nonlinearities.

Brochard and De Gennes predicted that doping of ferromagnetic particles to the LCs increases the response of the medium to the magnetic fields [26], which was experimentally confirmed [27]. Similarly, the LCs doped with ferroelectric nanoparticles (FNPs) were investigated experimentally by Reznikov et al. for the first time. Doping the LCs with FNPs increases the sensitivity of LCs to the electric field and their electro-optical performance $[28,29]$. Shelestiuk et al. calculated the dielectric permittivity and developed a theoretical model for the mentioned suspension [30]. The Freedericksz transition (FT) threshold of FNP doped smectic A LCs was investigated [31]. Lopatina and Selinger showed that FNPs can increase the isotropic-nematic phase transition temperature [32]. Also, we recently extended a theoretical model for the study of the spherical FNPs doped

*corresponding author; e-mail: jpoursamad@yahoo.com
NLCs in the presence of an electric field [33]. It was demonstrated that photorefractive effect is improved in the LC-FNP suspensions [34].

Due to the large permanent polarization of the FNPs, these nanoparticle tend to aggregate and chain together, and prohibition of this effect is difficult, experimentally $[28,29]$. Most of the studies ignore the aggregation effect of FNPs in these suspensions. Osipov and Gorkunov investigated the effect of FNPs chain formation on dielectric anisotropy and birefringence of the LC-FNP suspensions [35].

In this paper, optical nonlinearity of the LC-FNP suspension is studied for the chained spherical nanoparticles. Optical FT threshold of the suspension is compared with the pure $\mathrm{LC}$ and director reorientation and variations of the refractive index of the suspension are investigated for the oblique incidence of a light beam to the LC cell, in the small director reorientation regime. Also, the director reorientation is studied in the simultaneous presence of a light beam and an external magnetic field.

\section{Theory}

At optical frequencies, permanent dipoles of FNPs have minor impact on the suspension polarization, and the polarization is mainly due to light-induced dipoles in the medium. Characteristic times of orientational fluctuation of permanent dipoles are much larger than the inverse optical frequency. Therefore they have a minor contribution in the polarization [35]. Osipov and Gorkunov found the permittivity of the suspension as follows:

$$
\frac{\hat{\varepsilon}-1}{\hat{\varepsilon}+2}=\frac{4 \pi}{3}\left(\left\langle\hat{\beta}_{m}\right\rangle \rho_{m}+\left\langle\hat{\beta}_{n p}\right\rangle \rho_{n p}+\sum_{n=2}^{\infty}\left\langle\hat{\beta}_{n}\right\rangle \rho_{n}\right),
$$

where $\hat{\varepsilon}, \hat{\beta}$ and $\rho$ represent the permittivity tensor of the suspension, polarizability, and microscopic number density, respectively. The first, second, and third terms return to the LC molecules, nanoparticles and nanoparticles chains, respectively. One can obtain the permittivity 
by using $\left\langle\hat{\beta}_{n p \|}\right\rangle=m \beta+4 \beta^{2} S_{N P}(m-1) / \sigma^{3}$ and $\left\langle\hat{\beta}_{n p \perp}\right\rangle=$ $m \beta-2 \beta^{2} S_{N P}(m-1) / \sigma^{3}$ for the average polarizability of the nanoparticles chain parallel and perpendicular to the director, respectively. In this relations $m$, $\beta=1 / 8 \sigma^{3}\left(\varepsilon_{n p}-\varepsilon_{\perp}\right) /\left(\varepsilon_{n p}+2 \varepsilon_{\perp}\right), \sigma, \varepsilon_{n p}, \varepsilon_{\perp}$, and $S_{N P}$ are the number of the FNPs in a chain, polarizability of a FNP in the LC host, diameter of the FNPs, permittivity of the FNPs, permittivity of the LC host perpendicular to the director and order parameter of the FNPs, respectively. For using this relations one has to know the number of FNPs in a chain. We used the following approximations for calculating the suspension permittivities perpendicular and parallel to the LC director

$$
\begin{aligned}
& \frac{\varepsilon_{\perp}-1}{\varepsilon_{\perp}+2} \approx \frac{4 \pi}{3}\left(\left\langle\hat{\beta}_{m \perp}\right\rangle \rho_{m}+\left\langle\hat{\beta}_{n p \perp}\right\rangle \rho_{n p}\right), \\
& \frac{\varepsilon_{\|}-1}{\varepsilon_{\|}+2} \approx \\
& \quad \frac{4 \pi}{3}\left(\left\langle\hat{\beta}_{m \|}\right\rangle \rho_{m}+\left\langle\hat{\beta}_{n p \|}\right\rangle \rho_{n p}+\sum_{n=2}^{\infty}\left\langle\Delta \hat{\beta}_{n}\right\rangle \rho_{n}\right),
\end{aligned}
$$

where we ignored the chain polarization perpendicular to the director. However, the polarizabilities of the individual nanoparticles were taken into account. By using the Clausius-Mossetti relation we found 0.092, 0.072 , and $0.08 \mathrm{~cm}^{3}$ for the parallel and perpendicular polarizabilities of the mesogenic molecules and FNPs, respectively [36]. 5CB NLC parameters are used in the calculations $\varepsilon_{\|} \approx 2.9, \varepsilon_{\perp} \approx 2.3$ and $\varepsilon_{n p} \approx 5.76$ for the $\mathrm{BaTio}_{3}$ FNPs.

With the assumption of a moderate anisotropy of molecular polarizability $(\Delta \beta)$, the composite permittivity anisotropy is found to be as $\Delta \varepsilon=\Delta \varepsilon_{L C}+\Delta \varepsilon_{c h}$, where $\Delta \varepsilon_{L C}$ and $\Delta \varepsilon_{c h}$ are LC and FNPs chain anisotropies, respectively. $\Delta \varepsilon_{c h}$ was calculated as

$$
\Delta \varepsilon_{c h}=\frac{\pi}{24}\left[\frac{\left(\varepsilon_{\perp}+2\right)\left(\varepsilon_{n p}-\varepsilon_{\perp}\right)}{\varepsilon_{n p}+2 \varepsilon_{\perp}}\right]^{2} S_{N P} \rho^{*} \delta \eta .
$$

One can easily show that more accurate result is as follows:

$$
\Delta \varepsilon_{c h}=\frac{\pi}{24}\left(\varepsilon_{\perp}+2\right)\left(\varepsilon_{\|}+2\right)\left(\frac{\varepsilon_{n p}-\varepsilon_{\perp}}{\varepsilon_{n p}+2 \varepsilon_{\perp}}\right)^{2} S_{N P} \rho^{*} \delta \eta .
$$

In this formula $\varepsilon_{\|}, \varepsilon_{\perp}$, and $S_{N P}$ are LC parallel and perpendicular permittivities and NPs order parameter, respectively. $\rho^{*}=\rho \sigma^{3}$, where $\rho$ is the molar fraction of the FNPs and

$$
\begin{aligned}
& \delta \eta=2+\frac{1}{\eta}-\frac{4 \eta}{(\sqrt{1+4 \eta}-1)^{2}}, \\
& \eta=\pi \rho^{*} \mathrm{e}^{2 \lambda} /\left(18 \lambda^{3}\right),
\end{aligned}
$$

$\lambda$ is the FNPs dipole-dipole interaction strength. In Ref. [35] the order parameter of FNPs is considered to be equal to the LC order parameter. Lopatina and Selinger calculated the FNPs order parameter as

$$
S_{N P}=1-\frac{k_{\mathrm{B}} T}{K_{N P} S_{L C}},
$$

for the case that $K_{N P}$ is larger than $5 k_{\mathrm{B}} T$, where $K_{N P}=4 \pi \Delta \varepsilon_{L C} p^{2} / 45 \varepsilon^{2} R^{3}, T$ and $k_{\mathrm{B}}$ are temperature and the Boltzmann constant, respectively. $R$ and $p$ are the radius and permanent polarization of the FNPs.

We consider a homeotropic nematic LC cell and a laser light, which propagates through the cell.

Figure 1 demonstrates a homeotropic NLC cell doped with the spherical FNPs. The FNPs are aggregated and chained as shown. A laser light beam propagates with angle $\alpha$ with respect to the director initial orientation. $\theta$ indicates the director reorientation angle.

For the normal incidence of the laser light, there is a FT threshold for the director reorientation known as optical FT threshold as follows:

$$
E_{t h}=\frac{\pi}{L} \sqrt{\frac{4 \pi K}{\Delta \varepsilon}},
$$

whereas, there is no FT threshold for the oblique incidence of the light beam to the cell. When the light impinges with angle $\alpha$ with respect to the director initial direction, the torque balance equation will be as follows [37]:

$$
K \frac{\mathrm{d}^{2} \theta}{\mathrm{d} z^{2}}+\frac{\Delta \varepsilon\left\langle E_{o p}^{2}\right\rangle}{8 \pi} \sin 2(\theta+\alpha)=0,
$$

where $\theta, K, E_{o p}$ are the director reorients form the initial direction, the Frank elastic constant (ignoring the anisotropy of the elasticity), and optical field strength, respectively. In the small reorientation angle approximation, the equation can be written as

$$
2 \xi^{2} \frac{\mathrm{d}^{2} \theta}{\mathrm{d} z^{2}}+(2 \cos 2 \alpha) \theta+\sin 2 \alpha=0,
$$

where $\xi^{2}=4 \pi K /\left(\Delta \varepsilon\left\langle E_{o p}^{2}\right\rangle\right)$.

Solution of Eq. (10) depends on the sign of the second term's coefficient of and can be solved in two regimes. When this term is positive, one can obtain

$$
\theta=C_{1} \cos (\sqrt{ } \eta z)+C_{2} \sin (\sqrt{ } \eta z)-\lambda / \eta
$$

assuming the rigid anchoring conditions between the LC molecules and the cell boundaries, where $\eta=\cos 2 \alpha / \xi^{2}, \quad \lambda=\sin 2 \alpha / \xi^{2}, \quad C_{1}=\lambda / \eta, \quad$ and $C_{2}=(\lambda(1-\cos (\sqrt{\eta} L))) /(\eta \sin (\sqrt{\eta} L))$.

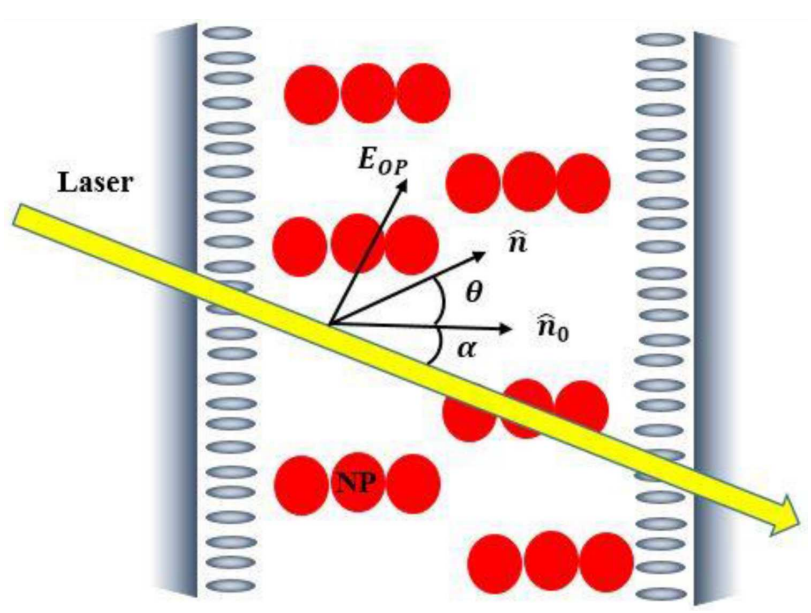

Fig. 1. Schematic illustration of the oblique propagation of the light beam from the LC cell. 
When the coefficient of the second term is negative, the same boundary conditions result in

$$
\theta=D_{1} \mathrm{e}^{\sqrt{-\eta} z}+D_{2} \mathrm{e}^{-\sqrt{-\eta} z}-\lambda / \eta
$$

where $D_{1}=\left(\lambda\left(1-\mathrm{e}^{-\sqrt{-\eta} L}\right)\right) /\left(\eta\left(\mathrm{e}^{\sqrt{-\eta} L}-\mathrm{e}^{-\sqrt{-\eta} L}\right)\right)$ and $D_{2}=\left(\lambda\left(\mathrm{e}^{\sqrt{-\eta} L}-1\right)\right) /\left(\eta\left(\mathrm{e}^{\sqrt{-\eta} L}-\mathrm{e}^{-\sqrt{-\eta} L}\right)\right)$.

An extraordinary wave experiences a refractive index change given by

$$
\Delta n=n_{e}(\theta+\alpha)-n_{e}(\alpha),
$$

where

$$
n_{e}(\alpha)=\sqrt{\frac{\varepsilon_{\|} \varepsilon_{\perp}}{\varepsilon_{\|} \cos ^{2}(\alpha)+\varepsilon_{\perp} \sin ^{2}(\alpha)}}
$$

The change in the refractive index in an NLC cell with the thickness of $L$ is

$$
\Delta n=\frac{1}{L} \int_{0}^{L}\left(n_{e}(\theta+\alpha)-n_{e}(\alpha)\right) \mathrm{d} z=\left\langle n_{2}\right\rangle I,
$$

where $I$ is the light intensity and $n_{2}$ characterizes the optical nonlinearity strength. In the small angle approximation, one can obtain

$$
\Delta n=\left\langle n_{2}\right\rangle I=\frac{\Delta \varepsilon \sqrt{\varepsilon_{\perp}}}{\varepsilon_{\|} L} \int_{0}^{L} \theta \mathrm{d} z,
$$

which for the first and second cases results in

$$
\begin{aligned}
\Delta n & =\left\langle n_{2}\right\rangle I=\frac{\Delta \varepsilon \sqrt{\varepsilon_{\perp}}}{\varepsilon_{\|} L} \\
\times & {\left[\frac{C_{1} \sin (\sqrt{\eta} L)-C_{2}(\cos (\sqrt{\eta} L)-1)}{\sqrt{\eta} L}-\frac{\lambda}{\eta}\right] } \\
\Delta n & =\left\langle n_{2}\right\rangle I=\frac{\Delta \varepsilon \sqrt{\varepsilon_{\perp}}}{\varepsilon_{\|} L} \\
& \times\left[\frac{D_{1}\left(\mathrm{e}^{\sqrt{-\eta} L}-1\right)-D_{2}\left(\mathrm{e}^{-\sqrt{-\eta} L}-1\right)}{\sqrt{-\eta} L}-\frac{\lambda}{\eta}\right],
\end{aligned}
$$

respectively. When an external magnetic field perpendicular to the initial director is applied, the coefficient of the second term in Eq. (10) changes to $2 \cos 2 \alpha+8 \pi \chi_{a} H^{2} / \Delta \varepsilon\left\langle E_{o p}^{2}\right\rangle$ and other parts of the equation remain unchanged. As in the previous case, solutions to Eq. (10) depend on the sign of this term, and have the same functional forms.

\section{Result and discussion}

It is essential to note that the permittivity of the FNPs in the optical frequencies is very different from the permittivity of these materials in the low frequencies. In the latter case, their permittivity is $3-5$ times larger than the in the former case. In the high frequency regime the permanent polarization of the FNPs is not taken into account, so the role of this polarization is not considered in this study. However, it is necessary to mention that the chain formation is due to the permanent polarization of the FNPs.
We found $\varepsilon_{\|} \approx 3.16$ and $\varepsilon_{\perp} \approx 2.3$ for parallel and perpendicular components of the suspension permittivity assuming $\rho=0.01$ and $R=30 \mathrm{~nm}$. These calculations show approximately 0.1 increase in the birefringence $\left(n_{e}-n_{o}\right)$ of the medium compared with the pure LC. $5 \mathrm{CB}$ NLC parameters are used in the calculations $\varepsilon_{\|} \approx 2.9, \varepsilon_{\perp} \approx 2.3, K=7 \times 10^{-7} \mathrm{dyn}, L=100 \mu \mathrm{m}$, and $\alpha=44^{\circ}$.

Optical FT threshold fields for the pure LCs and doped LCs are shown in Fig. 2 for the normal propagation of the light beam. The increase of the cell thickness reduces the threshold for the doped and pure LCs. The FT threshold of the doped LC is smaller than the FT threshold of the pure LC. It indicates that doping FNPs can affect the FT threshold of the LCs, significantly.

Changes of the refractive index $(\Delta n)$ for the pure LCs and doped LCs cells are indicated in Fig. 3. The optical field strengths are scaled by the optical FT threshold $E_{t h}$ of the pure LC. $\Delta n$ increases for the both the pure and doped NLCs. However, Fig. 3 shows that the doped NLC is a better medium for the optical nonlinearity observation. Its nonlinearity is approximately twice larger than the nonlinearity of the pure LC. $\left\langle n_{2}\right\rangle$ is roughly $1.1 \mathrm{~cm}^{2} / \mathrm{W}$ for the doped NLC and $0.6 \mathrm{~cm}^{2} / \mathrm{W}$ for the pure NLC. Therefore, the optical nonlinearity is in a range, which is named supra-nonlinearity [37].

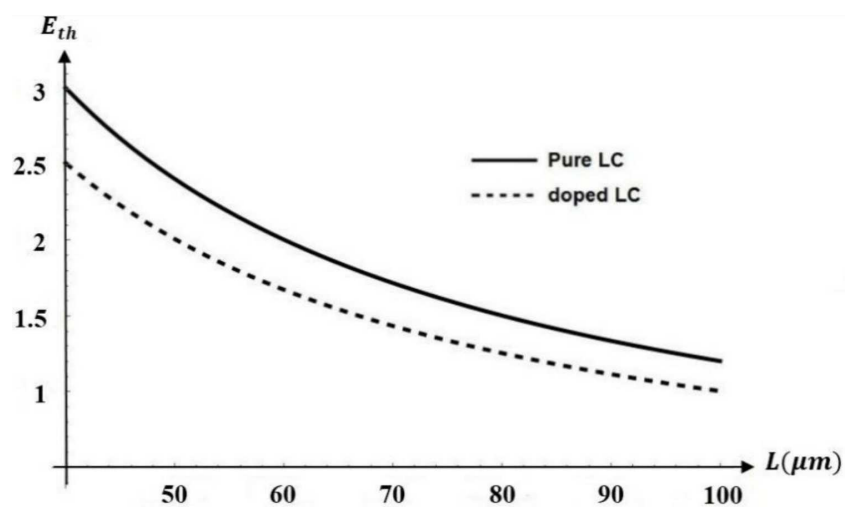

Fig. 2. Optical FT threshold with respect to the cell thickness.

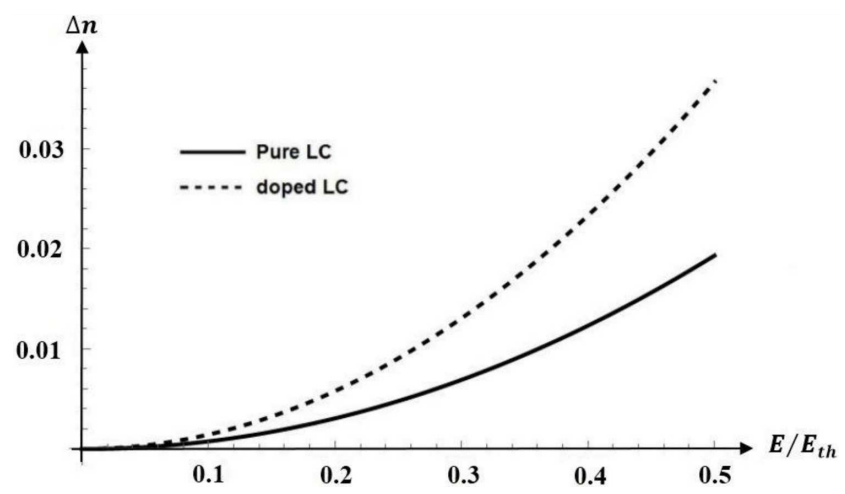

Fig. 3. Optical nonlinearity of the medium with respect to the optical field strength. 


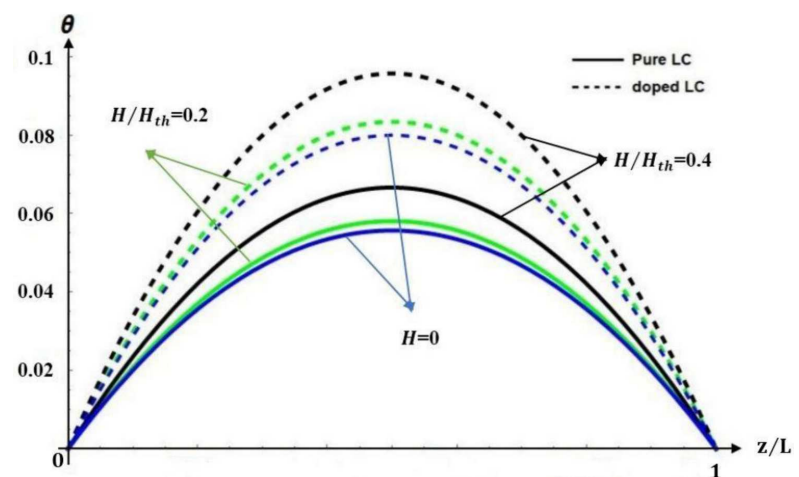

Fig. 4. Director reorientation for the pure and doped NLCs with and without external magnetic fields.

These calculations are done in the small director reorientation angle approximation. However, it must be noted that director reorientations larger than this limit can occur and lager experimental nonlinearities can be reached.

As mentioned earlier, optical nonlinearity in LCs is mainly due to the director reorientation. Most of the mechanisms of optical nonlinearities in the LCs are methods which transfer the beam energy to the director reorientation. Figure 4 compares the director reorientation for the doped LCs and pure LCs, in the presence and absence of an external magnetic field, assuming rigid anchoring conditions at the cell boundaries. As shown in Fig. 4, the director reorientation for the doped LC is larger than the pure LC for the both cases; presence or absence of a magnetic field. This larger director reorientation is the source of larger optical nonlinearity of the doped LCs.

Figure 4 indicates that when an external magnetic field is used the director reorientation increases and a probe beam experiences a larger phase shift. Increase of the applied magnetic field increases the director reorientation and consequently the phase shift experienced by a probe beam. Demonstrated magnetic fields scaled by the magnetic FT threshold $\left(H_{t h}=(\pi / L) \sqrt{K / \chi_{a}}\right)$, where $\chi_{a}$ is the magnetic anisotropy of the nematics.

\section{Conclusion}

Electric permittivities of an NLC cell doped with chained spherical FNPs parallel and perpendicular to the LC molecules are calculated. Optical FT threshold of the doped and pure LCs are compared with each other. Analytical solutions for the director reorientation of the LC molecules are found in the oblique incidence of a light beam, in the small director reorientation approximation. Sensed optical nonlinearity by an extraordinary wave is calculated, which is in the supra-nonlinearity range. It was shown that doping the nanoparticles increases the birefringence and optical nonlinearity of the host LC. Applying an external magnetic field, simultaneously with the light beam, increases the director reorientation in the $\mathrm{LC}$ cell.

\section{References}

[1] I.W. Stewart, The Static and Dynamic Theory of Liquid Crystals, Taylor and Francis, London 2004.

[2] R.H. Chen, Liquid Crystal Displays, Wiley, New Jersey 2011.

[3] S.D. Durbin, S.M. Arakclian, Y.R. Shen, Phys. Rev. Lett. 47, 1411 (1981).

[4] I.C. Khoo, Phys. Rev. A 23, 2077 (1981).

[5] I.C. Khoo, Phys. Rev. A 27, 2747 (1983).

[6] I.C. Khoo, S.L. Zhuang, Appl. Phys. Lett. 37, 3 (1980).

[7] I.C. Khoo, Phys. Rev. A 25, 1636 (1982).

[8] B.Ya. Zeldovich, N.F. Pilipetski, A.V. Sukhov, N.V. Tabiryan, Pis'ma Zh. Eksp. Teor. Fiz. 31, 287 (1980).

[9] I. Janossy, L. Csillag, A.D. Lloyd, Phys. Rev. A 44, 8410 (1991).

[10] I. Janossy, Phys. Rev. E 94, 2957 (1994).

[11] L. Vicari, F. Bloisi, F. Simoni, Appl. Phys. B 53, 314 (1991).

[12] I. Jánnosy, M.R. Taghizadeh, J. Gordon, H. Mathew, S. Desmond-Smith, IEEE J. Quant. Electron. 21, 1447 (1985)

[13] I.C. Khoo, Y. Liang, Phys. Rev. E 62, 6722 (2000).

[14] S.G. Odulov, Yu.A. Reznikov, M.S. Soskin, A.I. Khizhniak, Sov. Phys. JETP 55, 854 (1982).

[15] R. Elschner, R. Macdonald, J. Eichler, S. Hess, A.M. Sonnet, Phys. Rev. E 60, 1792 (1999).

[16] H.J. Eichler, R. Macdonald, Phys. Rev. Lett. 67, 2666 (1991)

[17] J.B. Poursamad, F.B. Jahanbakhsh, M. Asadpour, A. Phirouznia, J. Mol. Liq. 186, 23 (2013).

[18] J.B. Poursamad, F.B. Jahanbakhsh, M. Aas, K.M. Aghdami, M. Sahrai, R.S. Hakobyan, Mol. Cryst. Liq. Cryst. 560, 57 (2012).

[19] O. Francescangeli, S. Slussarenko, F. Simoni, D. Andrienko, V. Reshetnyak, Y. Reznikov, Phys. Rev. Lett. 82, 1855 (1999).

[20] E. Ouskova, Y. Reznikov, S.V. Shiyanovskii, L. Su, J.L. West, O.V. Kuksenok, O. Francescangeli, F. Simoni, Phys. Rev. E 64, 051709 (2001).

[21] K. Ichimura, Chem. Rev. Washington D.C. 100, 1847 (2000).

[22] J.B. Poursamad, A.K. Aleksanyan, R.S. Hakobyan, J. Appl. Phys. 108, 123113 (2010).

[23] J.B. Poursamad, Optik 127, 4872 (2016).

[24] J.B. Poursamad, A. Phirouznia, M. Sahrai, Physica B 476, 6 (2015).

[25] I.C. Khoo, M.Y. Shih, A. Shishido, P.H. Chen, M.V. Wood, Opt. Mater. 18, 85 (2001).

[26] F. Brochard, P.G. De Gennes, J. Phys. (France) 31, 691 (1970).

[27] S.H. Chen, N.M. Amer, Phys. Rev. Lett. 51, 2298 (1983).

[28] Y. Reznikov, O. Buchnev, O. Tereshchenko, V.Y. Reshetnyak, A. Glushchenko, Appl. Phys. Lett. 82, 1917 (2003). 
[29] E. Ouskova, O. Buchnev, V. Reshetnyak, Y. Reznikov, H. Kresse, Liq. Cryst. 30, 1235 (2003).

[30] S.M. Shelestiuk, V.Y. Reshetnyak, T.J. Sluckin, Phys. Rev. E 83, 041705 (2011).

[31] J.B. Poursamad, T. Hallaji, Physica B 504, 112 (2017).

[32] L.M. Lopatina, J.V. Selinger, Phys. Rev. Lett. 102, 197802 (2009).

[33] M. Emdadi, J.B. Poursamad, M. Sahrai, F. Moghaddas, Mol. Phys. 116, 1650 (2018).
[34] G. Cook, A.V. Glushchenko, V. Reshetnyak, A.T. Griffith, M.A. Saleh, D.R. Evans, Opt. Exp. 16, 4015 (2008).

[35] M.A. Osipov, M.V. Gorkunov, Phys. Rev. E 92 , 032501 (2015).

[36] T. Scharf, Polarized Light in Liquid Crystals and Polymers, Wiley, New Jersey 2007.

[37] I.C. Khoo, Phys. Rep. 471, 221 (2009). 